\title{
An Alternative Method for Removing Carnivore Teeth for Aging
}

METODA WYJMOWANIA ZEBOW U CARNIVORA DO OZNACZANIA WIEKU

\author{
Gwilm S. JONES \& Koren M. DRISCOLL
}

\begin{abstract}
Jones G. S., \& Driscoll K. M., 1982: An alternative method for removing carnivore teeth for aging. Acta theriol., 27, 12: 157-158.

A method for removing carnivore canines is proposed. It has the advantages of causing minimal damage to the mandible, requiring a brief time for extraction, and being effective on uncleaned specimens.
\end{abstract}

[Dept. Biol., Northeastern Univ., Boston, Mass. 02115, USA]

A variety of methods have been used to age carnivores including sagittal crest development, teeth wear, baculum development, suture closure, and cementum or dentin rings (Boise, 1975; Marks \& Erickson, 1966; Wright \& Coulter, 1967). The incremental cementum or dentin rings are frequently used by Fish and Game Department in the United States, although some consider it to be "... tedious and costly..." (Douglas \& Strickland, 1980). It is the removal of teeth for this purpose with which we concern ourselves in this paper. Prior to the development of the technique described here, teeth of the small and medium sized carnivores (e.g., Martes pennanti and Canis latrans) trapped or hunted in New Hampshire and Massachusetts were removed in the following manner: the anterior $1 / 3$ of a mandible was cut off with a saw at the level of the pm 4 and thru the mandibular symphysis; the canine was then removed by boiling or by decalcifying the mandibular fragment. This results in an excessively damaged mandible, much data which could later be recovered for systematic or anatomical studies having been lost. Others have removed tooth samples by boiling the mandibles. This technique has the disadvantage of loosening all of the teeth which, again, alters the anatomy for future studies, even if teeth are glued into place.

We have aged, by incremental rings, 540 Canis latrans, Say, 1823, 852 Martes pennanti (Erxleben, 1777), 613 Lutra canadensis, (Schreber, 1778), and 45 Felis (Lynx) rufus (Schreber, 1777) from New Hampshire. It was our intent, with every specimen, to preserve the skull as completely as possible for future studies. We have found that the following method accomplishes this with little loss of time when compared to the boiling technique and with a savings of time and effort when compared to fragmenting the specimen. It has the further advantage of being the most effective means of extracting a tooth prior to the muscle being removed from the mandible. We emphasize that we never use boiling water.

During necropsy, the mandibles are opened as wide as possible without detaching them from the cranium; this is facilitated by cutting the masseter and temporalis muscles. The skin is then cleared from the forward end of the mandibles and the forward $2 / 3$ 's of the tongue removed. We used both a Dremel Variable Seed Mato Tool with a sawtooth bit (which we prefer) and a low speed dental drill with 
a $7 \mathrm{~mm}$ circular bit to make three cuts in the dentary: 1) along the labial aspect of the tooth, following its curvature, and extending the length of the tooth, 2) from the labial gap between the third incisor and canine most of the way to the ventral extent of the mandibular symphysis, and 3) from the lingual gap between the third incisor and canine postero-ventrally along the curvature of the tooth. The depth of these cuts should ideally stop just as the saw completes its passage through the bone or just short of that, thus not damaging the tooth. This degree of precision is readily gained through practice on several specimens. We have experienced minimal but most often no damage to the tooth. The skull is then placed in a pan of shallow water which covers the area of the canine; the water is heated but not boiled. This soaking process takes as little as one hour but may take longer depending upon the length of the root. Upon sufficient soaking, the tooth can be pulled with a pair of pliers. The tooth is removed by a straight pull. If the tooth does not come relatively easily, soaking is continued. Obviously, numerous skulls can be soaked simultaneously.

This technique was used for all specimens which had not been cleaned of muscle. If the skulls were cleaned of muscle and connective tissue and then placed in heated water, the canine could often be pulled without cutting. However, of those which were thus cleaned and soaked, it was still necessary to employ the cutting technique on all old adults.

Advantages to this technique are the minimal amount of damage to the mandible and tooth, the short period of time expended by the researcher (as little as three minutes per skull), and the fact that the skull does not have to be cleaned prior to tooth removal.

Acknowledgements: We thank Eric Orff, New Hampshire Fish and Game Department, James Cardoza, Massachusetts Division of Fisheries and Wildlife and John. O. Whitaker Jr., Indiana State University for critically reading the manuscript. This project was partially funded by a contract from New Hampshire Fish and Game Department.

\section{REFERENCES}

Boise C. M., 1975: Skull measurements as criteria for aging hishers. N. Y. Fish and Game J., 22: 32-37. - Douglas C. \& Strickland M. (eds.), 1980: Transactions of fisher workshop held at Leslie M. Frost Natural Resources Centre, Dorset Ontario. 21-23 Oct. 1979, 139 pp. - Marks S. A. \& Erickson, A. W. 1966: Age determination in the black bear. J. Wildl. Mgt., 30: 389-410. - Wright, P. L. \& Coulter M. W., 1967: Reproduction and growth in Maine fishers. J. Wildl. Mgt., 31: 70-87.

Accepted, October 2, 1981. 